\title{
Optimal Sobolev Type Inequalities in Lorentz Spaces
}

\author{
Daniele Cassani · Bernhard Ruf • Cristina Tarsi
}

Received: 11 September 2012 / Accepted: 7 December 2012

(C) Springer Science+Business Media Dordrecht 2013

\begin{abstract}
It is well known that the classical Sobolev embeddings may be improved within the framework of Lorentz spaces $L^{p, q}$ : the space $\mathcal{D}^{1, p}\left(\mathbb{R}^{n}\right), 1<p<n$, embeds into $L^{p^{*}, q}\left(\mathbb{R}^{n}\right), p \leq q \leq \infty$. However, the value of the best possible embedding constants in the corresponding inequalities is known just in the case $L^{p^{*}, p}\left(\mathbb{R}^{n}\right)$. Here, we determine optimal constants for the embedding of the space $\mathcal{D}^{1, p}\left(\mathbb{R}^{n}\right), 1<p<n$, into the whole Lorentz space scale $L^{p^{*}, q}\left(\mathbb{R}^{n}\right), p \leq q \leq \infty$, including the limiting case $q=p$ of which we give a new proof. We also exhibit extremal functions for these embedding inequalities by solving related elliptic problems.
\end{abstract}

Keywords Sobolev inequalities $\cdot$ Lorentz spaces $\cdot$ Best constants $\cdot$ Optimal embeddings $\cdot$ Critical growth $\cdot$ Elliptic equations

Mathematics Subject Classifications (2010) $46 \mathrm{E} 35 \cdot 35 \mathrm{~B} 65 \cdot 35 \mathrm{~B} 38$

D. Cassani

Dip. di Scienza e Alta Tecnologia, Università degli Studi dell'Insubria,

Via Valleggio 11, 22100 Como, Italy

e-mail: Daniele.Cassani@uninsubria.it

B. Ruf $(\bowtie) \cdot$ C. Tarsi

Dip. di Matematica "F. Enriques", Università degli Studi di Milano,

Via C. Saldini 50, 20133 Milano, Italy

e-mail: Bernhard.Ruf@unimi.it

C. Tarsi

e-mail: Cristina.Tarsi@unimi.it 


\section{Introduction}

Let $\mathcal{D}^{1, p}\left(\mathbb{R}^{n}\right)$ be the completion with respect to the norm $\|\nabla \cdot\|_{p}$ of smooth compactly supported functions in $\mathbb{R}^{n}, n \geq 2$ and $1<p<n$; then, the Sobolev inequality [21] reads as follows

$$
\|u\|_{p^{*}} \leq S_{n, p^{*}}\|\nabla u\|_{p}, \quad \forall u \in \mathcal{D}^{1, p}\left(\mathbb{R}^{n}\right)
$$

where $p^{*}=n p /(n-p)$ is the critical Sobolev exponent and $S_{n, p^{*}}$ is the best possible constant in Eq. 1 which was obtained by T. Aubin [3] and G. Talenti [24] and is given by

$$
S_{n, p^{*}}=\frac{1}{\sqrt{\pi} n^{1 / p}}\left(\frac{p-1}{n-p}\right)^{\frac{p-1}{p}}\left[\frac{\Gamma(1+n / 2) \Gamma(n)}{\Gamma(n / p) \Gamma(1+n-n / p)}\right]^{1 / n}
$$

Moreover, the best constant is attained by the following functions

$$
u_{a, b}(x)=\left(a+b|x|^{\frac{p}{p-1}}\right)^{-\frac{n-p}{p}}
$$

for positive normalizing constants $a, b \in \mathbb{R}$; furthermore, as a consequence of the Gidas-Ni-Nirenberg symmetry result [13], Eq. 3 turns out to be the unique family of extremals to Eq. 1.

The knowledge of the best constant $S_{n, p^{*}}$ is important in applications to PDEs as the building block for the quantization of energy (see e.g. [23, 26]) which is a key-ingredient to establish existence of solutions to nonlinear elliptic equations with nonlinearities in the critical growth range.

Inequality (1) yields the embedding $\mathcal{D}^{1, p}\left(\mathbb{R}^{n}\right) \hookrightarrow L^{p^{*}}\left(\mathbb{R}^{n}\right)$ which is sharp in the context of Lebesgue spaces, in the sense that no smaller Lebesgue space can replace $L^{p^{*}}$. However, it is well known from the work of Peetre [19], see also [25] and references therein, that this embedding can be improved within the framework of Lorentz spaces.

Lorentz spaces $L^{p, q}$ are scales of interpolation spaces between Lebesgue spaces $L^{p}$ and can be defined via the notion of spherically symmetric decreasing rearrangement. For a measurable function $u: \Omega \rightarrow \mathbb{R}^{+}$, let $u^{*}$ denote its decreasing rearrangement which is defined as the distribution function of the distribution function $\mu_{u}$ of $u$, namely

$$
u^{*}(s)=\left|\left\{t \in[0,+\infty): \mu_{u}(t)>s\right\}\right|=\sup \left\{t>0: \mu_{u}(t)>s\right\}, \quad s \in[0,|\Omega|]
$$

whereas the spherically symmetric rearrangement $u^{\#}(x)$ of $u$ can be defined as

$$
u^{\#}(x)=u^{*}\left(\omega_{n}|x|^{n}\right), \quad x \in \Omega^{\#}
$$

where $\Omega^{\#} \subset \mathbb{R}^{n}$ is the open ball with center in the origin which satisfies $\left|\Omega^{\#}\right|=|\Omega|$ and $\omega_{n}$ is the area of the unit sphere of $\mathbb{R}^{n}$. Then, we define the Lorentz space $L^{p, q}(\Omega)$ as

$$
L^{p, q}(\Omega):=\left\{u: \Omega \rightarrow \mathbb{R} \text { measurable } \mid\|u\|_{p, q}:=\left(\int_{0}^{\infty}\left(u^{*}(t) t^{1 / p}\right)^{q} \frac{d t}{t}\right)^{\frac{1}{q}}<\infty\right\}
$$


where the quantity $\|u\|_{p, q}$ is a quasi-norm which turns out to be equivalent to a real norm. One clearly has $L^{p, p}=L^{p}$ and furthermore, with respect to the second index, Lorentz spaces satisfy the following inclusions

$$
L^{p, q_{1}} \subset L^{p, q_{2}} \text {, if } 1 \leq q_{1}<q_{2} \leq \infty
$$

where for $q=\infty$, the so-called Marcinkiewicz or weak- $L^{p}$ space, we define

$$
\|u\|_{p, \infty}:=\sup _{t>0} t^{\frac{1}{p}} u^{*}(t)
$$

The above mentioned improvement of the standard Sobolev embedding says that

$$
\mathcal{D}^{1, p}\left(\mathbb{R}^{n}\right) \hookrightarrow L^{p^{*}, p}\left(\mathbb{R}^{n}\right) \varsubsetneqq L^{p^{*}, p^{*}}\left(\mathbb{R}^{n}\right)=L^{p^{*}}\left(\mathbb{R}^{n}\right)
$$

The importance of the embedding (6) lies in the fact that it is optimal in the context of rearrangement invariant spaces (see [5] and [11]), since no "better" rearrangement invariant quasinorm than the Lorentz quasinorm $\|\cdot\|_{p^{*}, p}$ can be substituted in the embedding inequality:

$$
\|u\|_{p^{*}, p} \leq S_{n, p^{*}, p}\|\nabla u\|_{p},
$$

in which the best constant

$$
S_{n, p^{*}, p}=\frac{p}{n-p} \frac{[\Gamma(1+n / 2)]^{\frac{1}{n}}}{\sqrt{\pi}}
$$

was obtained by A. Alvino in [2].

Note that in view of Eq. 4 we have also the following embeddings:

$$
\mathcal{D}^{1, p}\left(\mathbb{R}^{n}\right) \hookrightarrow L^{p^{*}, q}\left(\mathbb{R}^{n}\right) \text {, for all } q \text { with } p \leq q \leq \infty
$$

The aim of this note is to obtain the value of the best possible embedding constants for the embeddings (8) and to exhibit a corresponding family of extremals, which will be obtained by solving related elliptic problems. Hereby, the embedding into the weak $L^{p}$-space seems of particular interest.

As a byproduct, we also give a new proof of the well known results when $q=p$ and $q=p^{*}$. Moreover, connections with embeddings of Sobolev spaces into weighted Lebesgue spaces (see [17]) are discussed in Section 5.

Recently it has been proved that the embedding (6) is not even locally compact, see $[9,14,20,22]$. We show here that this is due to the remarkable fact that all the embeddings (8) are invariant under the following group actions

$$
u_{\varepsilon}(r)=T_{\varepsilon} u(r)=\varepsilon^{-\frac{n-p}{p}} u\left(\frac{r}{\varepsilon}\right)
$$

which leave invariant the Sobolev norm $\|\nabla u\|_{p}$ as well as the Lorentz norms $\|u\|_{p^{*}, q}, p \leq q \leq \infty$. As a consequence, none of the embeddings (8) can be locally compact. Nevertheless, we will show that all the best embedding constants in the embeddings (8) are attained, except in the limiting (and optimal) case $q=p$, see Remark 2 below. 


\section{Main Results}

We state our main results distinguishing the three cases $q=\infty, 1<p<q<\infty$ and the limiting case $q=p$. Hereafter we denote by $\mathcal{D}_{0}^{1, p}(\Omega)$ the closure in $\mathcal{D}^{1, p}\left(\mathbb{R}^{n}\right)$ of smooth functions with compact support in $\Omega \subseteq \mathbb{R}^{n}$.

The case $q=+\infty$

Theorem 1 Let $\Omega \subseteq \mathbb{R}^{n}$ and $1<p<n$. Then, the following inequality holds

$$
\|u\|_{p^{*}, \infty} \leq S_{n, p^{*}, \infty}\|\nabla u\|_{p}
$$

for any $u \in \mathcal{D}_{0}^{1, p}(\Omega)$, where

$$
S_{n, p^{*}, \infty}=n^{-\frac{1}{p}} \omega_{n}^{-\frac{1}{n}}\left(\frac{p-1}{n-p}\right)^{\frac{p-1}{p}}
$$

Furthermore, the constant $S_{n, p^{*}, \infty}$ is sharp for any domain, it is never achieved as long as $\Omega \subsetneq \mathbb{R}^{n}$ stays bounded, and attained when $\Omega=\mathbb{R}^{n}$.

Explicit radially symmetric extremal functions for inequality (10), for $\Omega=\mathbb{R}^{n}$, can be obtained as solutions of an elliptic equation as established in the following

Theorem 2 Let $\Omega=\mathbb{R}^{n}$ and $\left\{u_{r_{0}}\right\}_{r_{0} \in(0, \infty)}$ be a (radial) family of extremal functions for inequality (10). Then for each $r_{0} \in(0, \infty), u_{r_{0}}(r)$ satisfies (weakly) the equation

$$
\left\{\begin{array}{l}
-\frac{d}{d r}\left(\left|y^{\prime}\right|^{p-2} y^{\prime} r^{n-1}\right)=\left(n \omega_{n}\right)^{-\frac{p-1}{p}}\left(\frac{n-p}{p-1}\right)^{\frac{p-1}{p}} r_{0}^{\frac{n-p}{p}} \delta_{r_{0}}, \\
y^{\prime}(0)=0, \\
y\left(r_{0}\right)=\left(n \omega_{n}\right)^{-\frac{1}{p}}\left(\frac{p-1}{n-p}\right)^{\frac{p-1}{p}} r_{0}^{-\frac{n-p}{p}}
\end{array}\right.
$$

The unique solution to problem (12) is the truncated function

$$
y_{r_{0}}(r)=\left(n \omega_{n}\right)^{-\frac{1}{p}}\left(\frac{p-1}{n-p}\right)^{\frac{p-1}{p}} \begin{cases}r_{0}^{-\frac{n-p}{p}}, & 0 \leq r \leq r_{0} \\ r_{0}^{\frac{n-p}{p(p-1)}} r^{-\frac{n-p}{p-1}}, & r_{0} \leq r<\infty\end{cases}
$$

Remark 1 We observe that Eq. 12 is invariant under the action of the group (9); actually, the truncated functions $u_{r_{0}}$ are invariant under the action of same group, and

$$
u_{r_{0}}(r)=T_{r_{0}} u_{1}(r)=r_{0}^{-\frac{n-p}{p}} u_{1}\left(r / r_{0}\right)
$$

The case $p<q<\infty$

Theorem 3 Let $1<p<n$ and $p<q<\infty$. Then, the following inequality holds

$$
\|u\|_{p^{*}, q} \leq S_{n, p^{*}, q}\|\nabla u\|_{p}
$$


for any $u \in \mathcal{D}^{1, p}\left(\mathbb{R}^{n}\right)$, with

$$
S_{n, p^{*}, q}=n^{\frac{1}{q}-\frac{1}{p}} \omega_{n}^{-\frac{1}{n}} p^{\frac{1}{q}} q^{-\frac{1}{p}}(q-p)^{\frac{1}{p}-\frac{1}{q}} \frac{(p-1)^{1+\frac{1}{q}-\frac{2}{p}}}{(n-p)^{\frac{1}{q}-\frac{1}{p}+1}}\left[B\left(\frac{q}{q-p}, \frac{q(p-1)}{q-p}\right)\right]^{-\frac{1}{p}+\frac{1}{q}}
$$

where $B(s, t)$ denotes the Euler beta function. The constant $S_{n, p^{*}, q}$ is sharp and is achieved by the family of extremal functions

$$
\psi(x)=\left(a+b|x|^{\frac{(n-p)(q)-p)}{p(p-1)}}\right)^{-\frac{p}{q-p}}, \quad a, b>0
$$

which originate from solutions to the following Euler-Lagrange equation related to Eq. 14

$$
-\Delta_{p} u=c(n, p, q)|x|^{\frac{q}{p}(n-p)-n}|u|^{q-2} u, \quad c(n, p, q)=\frac{q}{p} \frac{(n-p)^{p}}{(p-1)^{p-1}}
$$

and given by

$$
\psi_{\lambda}(x)=\lambda^{\frac{1}{q-p}}\left(\lambda+|x|^{\frac{(n-p)(q-p)}{p(p-1)}}\right)^{-\frac{p}{q-p}}, \quad \lambda>0
$$

Remark 2 Note that for $p<q \leq p^{*}$, Eq. 17 is the Euler-Lagrange equation corresponding to the critical embedding $\mathcal{D}^{1, p} \subset L^{q}\left(\frac{d x}{|x|^{s}}\right), s=n-\frac{q}{p}(n-p)$, i.e. into a weighted Lebesgue space; these solutions and the corresponding best constants are in fact known, cf. [17]. See Section 5.1 for further details.

The case $q=p$

We treat this case by taking the limit $q \searrow p$ in Theorem 3. We will show:

Theorem 4 Let $q \searrow p$. Then

(i) the constants in Eq. 15 tend to

$$
S_{n, p^{*}, p}=\omega_{n}^{\frac{1}{n}} \frac{p}{n-p}
$$

which is the constant obtained in [2];

(ii) the limiting Euler-Lagrange equation is associated to the well-known Hardy inequality which is known to have no solution in any domain $\Omega \subseteq \mathbb{R}^{n}$.

\section{Proof of Theorem 1}

We first consider the case in which $\Omega=\mathbb{R}^{n}$. Thanks to the Polya-Szego principle, we may assume that $u$ is a radial non increasing function; furthermore, by standard density arguments, we may suppose that $u$ is sufficiently smooth. We begin by performing the following change of variable

$$
r=t^{-\frac{p-1}{n-p}}, \quad r=|x|
$$


and defining

$$
w(t):=\left(n \omega_{n}\right)^{\frac{1}{p}}\left(\frac{n-p}{p-1}\right)^{\frac{p-1}{p}} u\left(t^{-\frac{p-1}{n-p}}\right)
$$

Then, $w(t):[0, \infty) \rightarrow \mathbb{R}^{+}$is an increasing function, $w(0)=0$ and

$$
w^{\prime}(t)=-\left(n \omega_{n}\right)^{\frac{1}{p}}\left(\frac{n-p}{p-1}\right)^{-\frac{1}{p}} t^{-\frac{p-1}{n-p}-1} u_{r}\left(t^{-\frac{p-1}{n-p}}\right)
$$

Hence

$$
\begin{aligned}
\|\nabla u\|_{p}^{p} & =n \omega_{n} \int_{0}^{\infty}\left|u_{r}\right|^{p} r^{n-1} d r \\
& =n \omega_{n} \frac{p-1}{n-p} \int_{0}^{\infty}\left|u_{r}\left(t^{-\frac{p-1}{n-p}}\right)\right|{ }^{p} t^{-n \frac{p-1}{n-p}-1} d t \\
& =\int_{0}^{\infty}\left|w^{\prime}(t)\right|^{p} d t
\end{aligned}
$$

whereas, recalling that $u^{*}(s)=u^{\sharp}\left(\left(\frac{s}{\omega_{n}}\right)^{1 / n}\right)$

$$
\begin{aligned}
\|u\|_{p^{*}, \infty} & =\sup _{s \in(0, \infty)} u^{*}(s) s^{\frac{1}{p^{*}}} \\
& =\sup _{s \in(0, \infty)} u\left(\left(\frac{s}{\omega_{n}}\right)^{1 / n}\right) s^{\frac{1}{p^{*}}} \\
& =\omega_{n}^{\frac{n-p}{n p}} \sup _{r \in(0, \infty)} u(r) r^{\frac{n-p}{p}} \\
& =n^{-\frac{1}{p}} \omega_{n}^{-\frac{1}{n}}\left(\frac{p-1}{n-p}\right)^{\frac{p-1}{p}} \sup _{t \in(0, \infty)} \frac{w(t)}{t^{\frac{p-1}{p}}}
\end{aligned}
$$

Let us now estimate $w(t)$ as follows:

$$
w(t)=\int_{0}^{t} w^{\prime}(s) d s \leq\left\{\int_{0}^{t}\left|w^{\prime}(s)\right|^{p} d s\right\}^{\frac{1}{p}} t^{\frac{p-1}{p}} \leq\|\nabla u\|_{p} t^{\frac{p-1}{p}}
$$

where we have used Eq. 19. Hence, by Eq. 21

$$
\|u\|_{p^{*}, \infty} \leq n^{-\frac{1}{p}} \omega_{n}^{-\frac{1}{n}}\left(\frac{p-1}{n-p}\right)^{\frac{p-1}{p}}\|\nabla u\|_{p}=S_{n, p^{*}, \infty}\|\nabla u\|_{p}
$$

We next prove that the constant $S_{n, p^{*}, \infty}$ is sharp by exhibiting a family of extremal functions for inequality (10). Let

$$
w_{k}(t)=\left\{\begin{array}{l}
\frac{t}{k^{1 / p}}, 0 \leq t \leq k \\
k^{\frac{p-1}{p}}, t \geq k
\end{array}\right.
$$


that is,

$$
u_{k}(r)=\left(n \omega_{n}\right)^{-\frac{1}{p}}\left(\frac{p-1}{n-p}\right)^{\frac{p-1}{p}} \begin{cases}k^{\frac{p-1}{p}}, & 0 \leq r \leq k^{-\frac{p-1}{n-p}} \\ k^{-\frac{1}{p}} r^{-\frac{n-p}{p-1}}, & r \geq k^{-\frac{p-1}{n-p}}\end{cases}
$$

Then

$$
\left\|\nabla u_{k}\right\|_{p}^{p}=\int_{0}^{\infty}\left|w_{k}^{\prime}\right|^{p} d t=\int_{0}^{k} \frac{d t}{k}=1
$$

and

$$
\left\|u_{k}\right\|_{p^{*}, \infty}=n^{-\frac{1}{p}} \omega_{n}^{-\frac{1}{n}}\left(\frac{p-1}{n-p}\right)^{\frac{p-1}{p}} \sup _{t \in(0, \infty)} \frac{w(t)}{t^{\frac{p-1}{p}}}=n^{-\frac{1}{p}} \omega_{n}^{-\frac{1}{n}}\left(\frac{p-1}{n-p}\right)^{\frac{p-1}{p}}=S_{n, p^{*}, \infty}
$$

Next we prove the second part of Theorem 1 in which $\Omega \subset \mathbb{R}^{n}$ is a bounded domain (with sufficiently smooth boundary). Clearly inequality (10) follows since any function $u \in \mathcal{D}_{0}^{1, p}(\Omega)$ can be trivially extended by 0 outside $\Omega$, obtaining a function which belongs to $\mathcal{D}^{1, p}\left(\mathbb{R}^{n}\right)$. Nevertheless, we next give a direct proof of the inequality also in the case of a bounded domain, since the one dimensional reduction will be crucial in the proof of the sharpness; as a byproduct we will get that the best constant turns out to be domain independent.

Consider a radial, non increasing function belonging to $\mathcal{D}_{0}^{1, p}\left(B_{R}\right)$, and consider the change of variable

$$
r=R t^{-\frac{p-1}{n-p}}
$$

together with

$$
w(t):=\left(n \omega_{n}\right)^{\frac{1}{p}}\left(\frac{n-p}{p-1}\right)^{\frac{p-1}{p}} R^{\frac{n-p}{p}} u\left(R t^{-\frac{p-1}{n-p}}\right)
$$

Then $w(t):[1, \infty) \rightarrow \mathbb{R}^{+}$, is increasing, $w(1)=0$ and

$$
\|\nabla u\|_{p}=\int_{1}^{\infty}\left|w^{\prime}(t)\right|^{p} d t, \quad\|u\|_{p^{*}, \infty}=n^{-\frac{1}{p}} \omega_{n}^{-\frac{1}{n}}\left(\frac{p-1}{n-p}\right)^{\frac{p-1}{p}} \sup _{t \in(1, \infty)} \frac{w(t)}{t^{\frac{p-1}{p}}}
$$

so that, as in the proof of Theorem 1 ,

$$
w(t)=\int_{1}^{t} w^{\prime}(s) d s \leq\left\{\int_{1}^{t}\left|w^{\prime}(s)\right|^{p} d s\right\}^{\frac{1}{p}} t^{\frac{p-1}{p}} \leq\|\nabla u\|_{p}(t-1)^{\frac{p-1}{p}}
$$

and inequality (10) follows.

Let us now prove the sharpness of the constant $S_{n, p^{*}, \infty}$ for any bounded domain. Actually it is enough to prove the sharpness for radial domains: indeed, for any bounded domain $\Omega \subset \mathbb{R}^{n}$ there exist $R, R^{\prime}>0$ such that $B_{R} \subset \Omega \subset B_{R^{\prime}}$ (up to performing translations which is possible since the symmetrization operator commutes with translations), so that by means of extension arguments we may consider just the class of radial domains. 
Thus set $\Omega=B_{R}$ and define

$$
w_{k}(t)=\left\{\begin{array}{l}
\frac{t-1}{(k-1)^{1 / p}}, 1 \leq t \leq k \\
(k-1)^{\frac{p-1}{p}}, t \geq k
\end{array}\right.
$$

that is,

$$
u_{k}(r)=\left(n \omega_{n}\right)^{-\frac{1}{p}}\left(\frac{p-1}{n-p}\right)^{\frac{p-1}{p}}\left\{\begin{array}{l}
(k-1)^{\frac{p-1}{p}}, \quad 0 \leq r \leq R k^{-\frac{p-1}{n-p}} \\
\frac{(R / r)^{\frac{n-p}{p-1}}-1}{(k-1)^{\frac{1}{p}}}, R k^{-\frac{p-1}{n-p}} \leq r \leq R
\end{array}\right.
$$

Then

$$
\left\|\nabla u_{k}\right\|_{p}^{p}=\int_{1}^{\infty}\left|w_{k}^{\prime}\right|^{p} d t=\int_{1}^{k} \frac{d t}{k-1}=1
$$

and

$$
\begin{aligned}
\left\|u_{k}\right\|_{p^{*}, \infty} & =n^{-\frac{1}{p}} \omega_{n}^{-\frac{1}{n}}\left(\frac{p-1}{n-p}\right)^{\frac{p-1}{p}} \sup _{t \in(1, \infty)} \frac{w(t)}{t^{\frac{p-1}{p}}} \\
& =n^{-\frac{1}{p}} \omega_{n}^{-\frac{1}{n}}\left(\frac{p-1}{n-p}\right)^{\frac{p-1}{p}}\left(1-\frac{1}{k}\right)^{\frac{p-1}{p}} \rightarrow S_{n, p^{*}, \infty}, \quad \text { as } k \rightarrow \infty
\end{aligned}
$$

It remains to prove that the best constant is never achieved: this can be done by adapting the argument developed in the proof of Theorem 2 in [7] and which we next recall. Let $\left\{u_{k}\right\}$ be a normalized maximizing sequence; we may suppose that $u_{k} \rightarrow u$ weakly in $\mathcal{D}_{0}^{1, p}(\Omega),\left|\nabla u_{k}\right|^{p} \rightarrow \mu$ weakly in the sense of measure. By means of Lions' concentration-compactness principle [15], we have two possible alternatives for the sequence $u_{k}$ : the weal limit $u \neq 0$ and compactness occurs and hence the best constant is achieved or $u=0$ and $\mu=\delta_{x_{0}}$ the Dirac mass at a point $x_{0}$. We show that the second case occurs.

Thanks to the Polya-Szego inequality, we may restrict our attention to radial and nondecreasing normalized maximizing sequences; we may also assume that $u_{k}$ is radially decreasing and sufficiently smooth. If $\left\{w_{k}\right\}$ is the sequence obtained from $\left\{u_{k}\right\}$ via the change of variable (24), then

$$
w_{k}(1)=0, \quad w_{k}^{\prime}(t) \geq 0, \quad \int_{1}^{+\infty}\left(w_{k}^{\prime}(t)\right)^{p} d t=1
$$

and

$$
\sup _{t \in(1, \infty)} \frac{w_{k}(t)}{t^{\frac{p-1}{p}}} \rightarrow 1^{-}
$$

This implies that for any $\varepsilon>0$ there exists $k_{\varepsilon} \in \mathbb{N}$ and $t_{\varepsilon} \in(1,+\infty)$ such that

$$
w_{k_{\varepsilon}}\left(t_{\varepsilon}\right)>(1-\varepsilon) t_{\varepsilon}^{\frac{p-1}{p}}
$$

On the other hand, for any $A \in(1, t)$ and for any $t>1$

$$
w(t)-w(A)=\int_{A}^{t} w^{\prime}(s) d s \leq\left\{\int_{A}^{t}\left(w^{\prime}\right)^{p} d s\right\}^{\frac{1}{p}}(t-A)^{\frac{p-1}{p}}
$$


so that, for any $\varepsilon>0$ and for any $A \in\left[1, t_{\varepsilon}\right)$

$$
(1-\varepsilon) t_{\varepsilon}^{\frac{p-1}{p}}<\left\{\int_{A}^{t_{\varepsilon}}\left(w_{k_{\varepsilon}}^{\prime}\right)^{p}\right\}^{\frac{1}{p}}\left(t_{\varepsilon}-A\right)^{\frac{p-1}{p}}+w_{k_{\varepsilon}}(A)
$$

Now choose $A=1$ in Eq. 26 to obtain $t_{\varepsilon} \longrightarrow \infty$ as $\varepsilon \rightarrow 0$. Furthermore, for any $A>$ 1 we infer

$$
\int_{1}^{A}\left(w_{k_{\varepsilon}}^{\prime}\right)^{p} d s \longrightarrow 0
$$

Indeed, if not, one has

$$
\int_{A}^{t_{\varepsilon}}\left(w_{k_{\varepsilon}}^{\prime}\right)^{2}<1-\delta
$$

for some $\delta>0$ and this contradicts Eq. 26 .

Therefore, up to a subsequence, $\left\{w_{k}\right\}$ concentrates at $+\infty$, so that the corresponding $\left\{u_{k}\right\}$ turns out to be a normalized maximizing sequence which concentrates at 0 .

\section{Elliptic Problems Related to Extremals: Proof of Theorem 2}

Let us now restrict our attention to a family of radial extremal functions to inequality (10) which originate from solving associated Euler-Lagrange equations. As previously observed, the Polya-Szego inequality implies that

$$
\left(n \omega_{n}\right)^{-1}\left(\frac{p-1}{n-p}\right)^{p-1}=\sup _{u \in \mathcal{D}_{0, r a d}^{1, p},\|\nabla u\|_{p}^{p}=1} \psi_{\#}(u)
$$

where

$$
\psi_{\#}(u)=\sup _{r \in(0, \infty)}\left|u^{\#}(r)\right|^{p} r^{n-p}=\omega_{n}^{-\frac{n-p}{n}}\|u\|_{p^{*}, \infty}^{p}
$$

and the supremum is attained if and only if the constant

$$
\left(n \omega_{n}\right)^{-1}\left(\frac{p-1}{n-p}\right)^{p-1}
$$

is achieved.

Now the idea is to derive the Euler-Lagrange equation related to Eq. 27. However, since the $L^{p^{*}, \infty}$ quasi-norm is not differentiable, we replace the optimization problem (27) by an equivalent one which involves a convex functional and which thus admits a nonempty subdifferential set.

Note that for any $u \in \mathcal{D}_{0, \text { rad }}^{1, p}\left(\mathbb{R}^{n}\right)$ one has

$$
\begin{aligned}
|u(r)| & =\left|\int_{r}^{+\infty} u^{\prime} d \rho\right| \leq\left(n \omega_{n}\right)^{-\frac{1}{p}}\left\{n \omega_{n} \int_{r}^{\infty}\left|u^{\prime}(\rho)\right|^{p} \rho^{n-1} d \rho\right\}^{\frac{1}{p}}\left\{\int_{r}^{\infty} \rho^{-\frac{n-1}{p-1}} d \rho\right\}^{\frac{p-1}{p}} \\
& \leq\left(n \omega_{n}\right)^{-\frac{1}{p}}\left(\frac{n-p}{p-1}\right)^{\frac{p-1}{p}}\|\nabla u\|_{p} r^{-\frac{n-p}{p}}
\end{aligned}
$$


so that

$$
\psi(u):=\sup _{r \in(0, \infty)}|u(r)|^{p} r^{n-p} \leq\left(n \omega_{n}\right)^{-1}\left(\frac{p-1}{n-p}\right)^{p-1}\|\nabla u\|_{p}^{p}
$$

and thus

$$
\left(n \omega_{n}\right)^{-1}\left(\frac{p-1}{n-p}\right)^{p-1} \geq \sup _{u \in \mathcal{D}_{0, r a d}^{1, p},\|\nabla u\|_{p}^{p}=1} \psi(u)
$$

On the other hand,

$$
\psi_{\#}(u)=\sup _{r \in(0, \infty)}|u(r)|^{p} r^{n-p}=\psi(u) \quad \text { for any } \quad u=u^{\#}
$$

and since via the Polya-Szego inequality there exist positive and radially decreasing extremal functions to inequality (10), we can conclude that

$$
\left(n \omega_{n}\right)^{-1}\left(\frac{p-1}{n-p}\right)^{p-1}=\sup _{u \in \mathcal{D}_{0, \text { rad }}^{1, p},\|\nabla u\|_{p}^{p}=1} \psi(u)
$$

which implies directly

$$
\sup _{u \in \mathcal{D}_{0, \text { rad }}^{1, p},\|\nabla u\|_{p}^{p}=1} \psi_{\sharp}(u)=\sup _{u \in \mathcal{D}_{0, \text { rad }}^{1, p},\|\nabla u\|_{p}^{p}=1} \psi(u)
$$

Furthermore, the extremal functions to Eq. 27 satisfy the Euler Lagrange ineqality associated to Eq. 30. Notice that though the functional $u \mapsto \psi(u)$ fails to be differentiable, it is convex and therefore its subdifferential $\partial \psi(u)$ is well defined.

For the convenience of the reader, let us briefly recall some basic definitions and properties of the subdifferential and the subgradient of a convex function:

Definition 1 Let $E$ be a Banach space, and $\psi: E \rightarrow \mathbb{R}$ continuous and convex. Then the subdifferential $\partial \psi(u)$ of $\psi$ at $u \in E$ is the subset of the dual space $E^{\prime}$ characterized by

$$
\eta_{u} \in \partial \psi(u) \Longleftrightarrow \psi(u+v)-\psi(u) \geq\left\langle\eta_{u}, v\right\rangle, \quad \forall v \in E
$$

where $\langle\cdot, \cdot\rangle$ denotes the duality pairing between $E$ and $E^{\prime}$. An element $\eta_{u} \in \partial \psi(u)$ is called a subgradient of $\psi$ at $u$.

Adapting the proofs of Lemmas 2.2 and 2.3 and Corollary 2.4 in [10] one has the following results

Lemma 1 Let $\psi: E \rightarrow \mathbb{R}$ be convex and continuous. Assume $\psi(x) \geq 0$ for all $x \in E$ and

$$
\psi(t x)=t^{q} \psi(x), \quad \forall t \geq 0
$$

where $q \geq 1$. Then

$$
\mu \in \partial \psi(u) \Longleftrightarrow\left\{\begin{array}{l}
\langle\mu, u\rangle=q \psi(u) \\
\langle\mu, x\rangle \leq\langle\mu, u\rangle, \forall x \in \psi^{u}:=\{x \in E: \psi(x) \leq \psi(u)\}
\end{array}\right.
$$


Lemma 2 Let $E$ be a Banach space and assume that $\phi \in \mathcal{C}^{1}(E ; \mathbb{R})$ satisfies

$$
\left\langle\phi^{\prime}(x), x\right\rangle=q \phi(x) \neq 0, \quad \forall x \in E \backslash\{0\}
$$

Let $\psi: E \rightarrow \mathbb{R}$ satisfy the hypotheses of Lemma 1 . If $y \in E$ is such that, for some $A>0$

$$
\psi(y)=\sup _{u \in E, \phi(u)=1} \psi(u)=\frac{1}{A}
$$

then

$$
\phi^{\prime}(y) \in \frac{1}{\psi(y)} \partial \psi(y) \equiv A \partial \psi(y)
$$

Specializing Lemmas 1 and 2 to our situation we obtain

Proposition 1 Let $E=\mathcal{D}_{0, \text { rad }}^{1, p}\left(\mathbb{R}^{n}\right)$ and consider $\psi(u)$ defined by Eq. 29. Suppose that $y \in E$ satisfies

$$
\psi(y)=\sup _{u \in E,\|\nabla u\|_{p}^{p}=1} \psi(u)=\frac{1}{n \omega_{n}}\left(\frac{p-1}{n-p}\right)^{p-1}
$$

Then y satisfies, in the weak sense, the equation

$$
\left\{\begin{array}{l}
-\frac{d}{d r}\left(\left|y^{\prime}\right|^{p-2} y^{\prime} r^{n-1}\right)=\frac{1}{p}\left(\frac{n-p}{p-1}\right)^{p-1} \mu_{y}, \text { where } \mu_{y} \in \partial \psi(y) \subset E^{\prime} \\
y^{\prime}(0)=0,
\end{array}\right.
$$

Proof of Proposition 1 By Lemma 2 we obtain that $y$ satisfies

$$
n \omega_{n} \int_{0}^{\infty} p\left|y^{\prime}\right|^{p-2} y^{\prime} v^{\prime} r^{n-1} d r=n \omega_{n}\left(\frac{n-p}{p-1}\right)^{p-1}\left\langle\mu_{y}, v\right\rangle, \quad \forall v \in E
$$

and the claim easily follows.

It remains to determine the subgradient $\mu_{y}$ in Eq. 31. Following the lines of Lemmas 2.6, 2.7 and 2.8 of [10] we get

Proposition 2 Let y be a maximizer for problem (30) and let

$$
K_{y}=\left\{r \in(0, \infty) \mid y^{p}(r) r^{n-p}=\sup _{\rho \in(0, \infty)} y^{p}(\rho) \rho^{n-p}=\frac{1}{n \omega_{n}}\left(\frac{p-1}{n-p}\right)^{p-1}\right\}
$$

Then

i) $\operatorname{supp}\left(\mu_{y}\right) \subset K_{y}, \quad \forall \mu_{y} \in \partial \psi(y)$;

ii) $\left\langle\mu_{y}, y\right\rangle=p \psi(y)=\frac{p}{n \omega_{n}}\left(\frac{p-1}{n-p}\right)^{p-1}$;

iii) $K_{y}=\left\{r_{0}\right\}$;

iv) $\partial \psi(y)=[\psi(y)]^{\frac{p-1}{p}} p r_{0}^{\frac{n-p}{p}} \delta_{r_{0}}=\frac{p}{\left(n \omega_{n}\right)^{\frac{p-1}{p}}}\left(\frac{p-1}{n-p}\right)^{\frac{(p-1)^{2}}{p}} r_{0}^{\frac{n-p}{p}} \delta_{r_{0}}$ 
Proof of Proposition 2 Statements i) and ii) follow by slight changes to the argument in [8] where the Moser limiting case $p=n$ is considered.

For proving iii) let us first prove that $\operatorname{supp}\left(\mu_{y}\right)$ does not contain intervals. By contradiction, suppose that $\operatorname{supp}\left(\mu_{y}\right)$ contains an interval $I=\left(r_{1}, r_{2}\right), 0 \leq r_{1}<r_{2}$ with

$$
y(r)=\left(n \omega_{n}\right)^{-\frac{1}{p}}\left(\frac{p-1}{n-p}\right)^{\frac{p-1}{p}} r^{-\frac{n-p}{p}}, \quad r \in I
$$

From Eq. 32 and ii) we obtain

$$
n \omega_{n} \int_{0}^{\infty}\left|y^{\prime}\right|^{p} r^{n-1} d r=\frac{n \omega_{n}}{p}\left(\frac{n-p}{p-1}\right)^{p-1}\left\langle\mu_{y}, y\right\rangle=1
$$

that is

$$
n \omega_{n} \int_{0}^{\infty}\left|y^{\prime}\right|^{p} r^{n-1} d r=1
$$

which is the constraint in the maximum problem (30). Performing the change of variable (18) and recalling that the $y(r)=y^{\#}(r)$ we obtain:

$$
w(t)=t^{\frac{p-1}{p}}, \quad \forall t \in\left(t_{1}, t_{2}\right) ; \quad 0 \leq t_{1}<t_{2} \leq \infty ; \quad \int_{0}^{\infty}\left|w^{\prime}\right|^{p} d t=1
$$

where $w(t)=\left(n \omega_{n}\right)^{\frac{1}{p}}\left(\frac{n-p}{p-1}\right)^{\frac{p-1}{p}} u\left(t^{-\frac{p-1}{n-p}}\right), t_{1}=r_{2}^{-\frac{n-p}{p-1}}$ and $t_{2}=r_{1}^{-\frac{n-p}{p-1}}$. Observe that

$$
\int_{t_{1}}^{t_{2}}\left|w^{\prime}\right|^{p} d t=\left(\frac{p-1}{p}\right)^{p} \int_{t_{1}}^{t_{2}} \frac{d t}{t}
$$

so that $0 \lesseqgtr t_{1}<t_{2} \lesseqgtr \infty$. Hence

$$
w(t)= \begin{cases}\varphi_{1}(t), & 0 \leq t \leq t_{1} \\ t^{\frac{p-1}{p}}, & t_{1} \leq t \leq t_{2} \\ \varphi_{2}(t), & t_{2} \leq t<\infty\end{cases}
$$

and letting $\delta=\int_{t_{1}}^{t_{2}}\left|w^{\prime}\right|^{p} d t \in(0,1)$ we have

$$
0<\int_{0}^{t_{1}}\left|w^{\prime}\right|^{p} d t=\int_{0}^{t_{1}}\left|\varphi_{1}^{\prime}\right|^{p} d t \leq 1-\delta
$$

A straightforward calculation shows that

$$
\inf \left\{\int_{0}^{t_{1}}\left|\varphi_{1}^{\prime}\right|^{p} d t, \varphi_{1}(t) \mathcal{C}^{1} \text {-piecewise }, \varphi_{1}\left(t_{1}\right)=t_{1}^{\frac{p}{p-1}}\right\}
$$

is achieved by a line and its value is 1 , thus a contradiction.

Actually in the above argument we have also proved that if $r_{0} \in K_{y}$, than $y$ is a truncated function, so that $K_{y}=\left\{r_{0}\right\}$. 
Finally to prove iv) notice that from $K_{y}=\left\{r_{0}\right\}$ we have

$$
\begin{aligned}
\psi(y+v) & =\sup _{r \in(0,1)}|y(r)+v(r)|^{p} r^{n-p} \geq\left|y\left(r_{0}\right)+v\left(r_{0}\right)\right|^{p} r_{0}^{n-p} \\
& \geq\left|y\left(r_{0}\right)\right|^{p} r_{0}^{n-p}+p\left[y\left(r_{0}\right)\right]^{p-1} v\left(r_{0}\right) r_{0}^{\frac{n-p}{p}} \\
& =\psi(y)+[\psi(y)]^{\frac{p-1}{p}} p v\left(r_{0}\right) r_{0}^{\frac{n-p}{p}}
\end{aligned}
$$

Hence $\mu_{y}=p[\psi(y)]^{\frac{p-1}{p}} \delta_{r_{0}} r_{0}^{\frac{n-p}{p}}$. Vice versa, let $\mu_{y}$ be an arbitrary element of $\partial y$. Since $\operatorname{supp}\left(\mu_{y}\right) \subset K_{y}=\left\{r_{0}\right\}$, one has $\operatorname{supp}\left(\mu_{y}\right)=\left\{r_{0}\right\}$; hence the distribution $\mu_{y}$ has finite order $N$ and can be represented as $\mu_{y}=\sum_{i=0}^{N} a_{1} D^{i} \delta_{r_{0}}$. But $\mu_{y} \in\left(\mathcal{D}_{0, \text { rad }}^{1, p}\right)^{\prime}$ so that $N=0$ and $\mu_{y}=a \delta_{r_{0}}$; recalling ii) the thesis follows.

Resuming the proof of Theorem 2 we have so far obtained

$$
\mu_{y}=p r_{0}^{\frac{n-p}{p}}\left(n \omega_{n}\right)^{-\frac{p-1}{p}}\left(\frac{p-1}{n-p}\right)^{\frac{(p-1)^{2}}{p}} \delta_{r_{0}}
$$

By Propositions 1 and 2 we infer that if $y$ is a maximizer for Eq. 30, then it satisfies (weakly) the equation

$$
\left\{\begin{array}{l}
-\frac{d}{d r}\left(\left|y^{\prime}\right|^{p-2} y^{\prime} r^{n-1}\right)=\left(n \omega_{n}\right)^{-\frac{p-1}{p}}\left(\frac{n-p}{p-1}\right)^{\frac{p-1}{p}} r_{0}^{\frac{n-p}{p}} \delta_{r_{0}} \\
y^{\prime}(0)=0
\end{array}\right.
$$

where

$$
y\left(r_{0}\right)=\left(n \omega_{n}\right)^{-\frac{1}{p}}\left(\frac{p-1}{n-p}\right)^{\frac{p-1}{p}} r_{0}^{-\frac{n-p}{p}}
$$

that is Eq. 12.

Let us now determine the solution to this problem: by definition, we have

$$
\int_{0}^{\infty}\left|y^{\prime}\right|^{p-2} y^{\prime} v^{\prime} r^{n-1}=\left(n \omega_{n}\right)^{-\frac{p-1}{p}}\left(\frac{n-p}{p-1}\right)^{\frac{p-1}{p}} r_{0}^{\frac{n-p}{p}} v\left(r_{0}\right)
$$

for any $v \in \mathcal{D}_{0, \text { rad }}^{1, p}\left(R^{n}\right)$. Let $v$ be smooth, with compact support, then

$$
\begin{aligned}
\left(n \omega_{n}\right)^{-\frac{p-1}{p}} & \left(\frac{n-p}{p-1}\right)^{\frac{p-1}{p}} r_{0}^{\frac{n-p}{p}} v\left(r_{0}\right) \\
=\lim _{\eta \rightarrow 0^{+}} & {\left[\int_{0}^{r_{0}-\eta}\left|y^{\prime}\right|^{p-2} y^{\prime} v^{\prime} r^{n-1} d r+\int_{r_{0}+\eta}^{\infty}\left|y^{\prime}\right|^{p-2} y^{\prime} v^{\prime} r^{n-1} d r\right] } \\
=\lim _{\eta \rightarrow 0^{+}} & {\left[\left|y^{\prime}\right|^{p-2} y^{\prime}\left(r_{0}-\eta\right) v\left(r_{0}-\eta\right)\left(r_{0}-\eta\right)^{n-1}-\int_{0}^{r_{0}-\eta} \frac{d}{d r}\left(\left|y^{\prime}\right|^{p-2} y^{\prime} r^{n-1}\right) v d r\right.} \\
& \left.\quad\left|y^{\prime}\right|^{p-2} y^{\prime}\left(r_{0}+\eta\right) v\left(r_{0}+\eta\right)\left(r_{0}+\eta\right)^{n-1}-\int_{r_{0}+\eta}^{\infty} \frac{d}{d r}\left(\left|y^{\prime}\right|^{p-2} y^{\prime} r^{n-1}\right) v d r\right]
\end{aligned}
$$


so that $y$ satisfies the problem

$$
\left\{\begin{array}{l}
-\frac{d}{d r}\left(\left|y^{\prime}\right|^{p-2} y^{\prime} r^{n-1}\right)=0 \quad r \in\left(0, r_{0}\right) \cup\left(r_{0}, \infty\right) \\
y^{\prime}(0)=0, \\
r_{0}^{n-1}\left[\left(y_{+}^{\prime}\left(r_{0}\right)\right)^{p-1}-\left(y_{-}^{\prime}\left(r_{0}\right)\right)^{p-1}\right]=\left(n \omega_{n}\right)^{-\frac{p-1}{p}}\left(\frac{n-p}{p-1}\right)^{\frac{p-1}{p}} r_{0}^{\frac{n-p}{p}}
\end{array}\right.
$$

and

$$
y\left(r_{0}\right)=\left(n \omega_{n}\right)^{-\frac{1}{p}}\left(\frac{p-1}{n-p}\right)^{\frac{p-1}{p}} r_{0}^{-\frac{n-p}{p}}
$$

Hence

$$
y_{r_{0}}(r)= \begin{cases}c_{1}, & 0 \leq r<r_{0} \\ c_{2} r^{-\frac{n-p}{p-1}}, & r_{0}<r<\infty\end{cases}
$$

where

$$
c_{1}=\left(n \omega_{n}\right)^{-\frac{1}{p}}\left(\frac{p-1}{n-p}\right)^{\frac{p-1}{p}} r_{0}^{-\frac{n-p}{p}}, \quad c_{2}=c_{1} r_{0}^{\frac{n-p}{p-1}}=\left(n \omega_{n}\right)^{-\frac{1}{p}}\left(\frac{p-1}{n-p}\right)^{\frac{p-1}{p}} r_{0}^{\frac{n-p}{p(p-1)}}
$$

that is Eq. 13.

\section{The Case $p<q<\infty$ : Proof of Theorem 3}

The key in the proof of Theorem 3 is a suitable change of variable which allows us to reduce the problem to the case treated in [24]. Thanks to the Polya-Szego inequality, finding the best constants $S_{n, p^{*}, q}$ is equivalent to maximize the ratio

$$
J_{n, q, p}(u):=n^{\frac{1}{q}-\frac{1}{p}} \omega_{n}^{-\frac{1}{n}} \frac{\left(\int_{0}^{\infty} u^{q} r^{\frac{q}{p}(n-p)-1} d r\right)^{\frac{1}{q}}}{\left(\int_{0}^{\infty}\left|u^{\prime}(r)\right|^{p} r^{n-1} d r\right)^{\frac{1}{p}}}
$$

where $u(r)$ is a positive decreasing function on $[0, \infty)$ sufficiently smooth (e.g. Lipschitz continuous) and such that $\int_{0}^{\infty}\left|u^{\prime}(r)\right|^{p} r^{n-1} d r<\infty$. Let us now perform the change of variable (see also [12], proof of Lemma 7)

$$
r=\rho^{\frac{p^{2}}{(n-p)(q-p)}}, \quad p<q<\infty
$$

and define

$$
v(\rho)=u\left(\rho^{\frac{p^{2}}{(n-p)(q-p)}}\right)
$$

Then $v(\rho)$ is still Lipschitz continuous on $(0, \infty)$, positive and decreasing, with

$$
\int_{0}^{\infty}\left|u^{\prime}(r)\right|^{p} r^{n-1} d r=\left(\frac{(q-p)(n-p)}{p^{2}}\right)^{p-1} \int_{0}^{\infty}\left|v^{\prime}(\rho)\right|^{p} \rho^{\frac{p q}{q-p}-1} d \rho
$$


and

$$
\int_{0}^{\infty} u^{q} r^{\frac{q}{p}(n-p)-1} d r=\frac{p^{2}}{(q-p)(n-p)} \int_{0}^{\infty} v(\rho)^{q} \rho^{\frac{p q}{q-p}-1} d \rho
$$

To conclude the proof we need the following Lemma due to Talenti

Lemma 3 (Lemma 2 in [24]) Let $m, p, q$ be real numbers such that

$$
1<p<m, \quad q=\frac{m p}{m-p}
$$

Let $u$ be any real valued function of a real variable $r$, which is sufficiently smooth on the half line $(0,+\infty)$ (e.g. Lipschitz continuous) and such that

$$
\int_{0}^{+\infty} r^{m-1}\left|u^{\prime}(r)\right|^{p} d r<+\infty, \quad u(r) \rightarrow 0, \quad \text { as } r \rightarrow+\infty
$$

Set

$$
I_{m, p}(u):=\frac{\left(\int_{0}^{\infty} r^{m-1}|u|^{q} d r\right)^{\frac{1}{q}}}{\left(\int_{0}^{\infty} r^{m-1}\left|u^{\prime}(r)\right|^{p} d r\right)^{\frac{1}{p}}}
$$

Then

$$
I_{m, p}(u) \leq I_{m, p}(\varphi)
$$

where $\varphi$ is any function of the form

$$
\varphi(r)=\left(a+b r^{\frac{p}{p-1}}\right)^{1-\frac{m}{p}}
$$

with $a$ and $b$ positive constants. A straightforward calculation yields

$$
I_{m, p}(\varphi)=m^{-\frac{1}{p}}\left(\frac{p-1}{m-p}\right)^{\frac{p-1}{p}}\left(\frac{p}{p-1}\right)^{\frac{1}{m}}\left[\frac{\Gamma(m)}{\Gamma\left(\frac{m}{p}\right) \Gamma\left(\frac{m(p-1)}{p}\right)}\right]^{\frac{1}{m}}
$$

The extremals are solutions of the differential equation

$$
\left(r^{m-1}\left|u^{\prime}\right|^{p-1} \operatorname{sgn}\left(u^{\prime}\right)\right)^{\prime}=m\left(\frac{m-p}{p-1}\right)^{p-1} a b^{p-1} r^{m-1}|u|^{q-1}
$$

verifying the conditions

$$
\int_{0}^{\infty} r^{m-1}\left|u^{\prime}(r)\right|^{p} d r<\infty, u(r) \rightarrow 0 \text { as } r \rightarrow+\infty, u^{\prime}(r)=o\left(r^{-\frac{m}{p}}\right) \text { as } r \rightarrow 0 \text { or }+\infty
$$

Conversely, any solution to Eq. 36 with the above properties is an extremal for $I_{m, p}$.

Now set

$$
m=\frac{p q}{q-p}
$$


so that $\frac{m p}{m-p}=q$ and recalling Eqs. 34 and 35, we have

$$
\begin{aligned}
J_{n, q, p}(u) & =n^{\frac{1}{q}-\frac{1}{p}} \omega_{n}^{-\frac{1}{n}}\left(\frac{p^{2}}{(q-p)(n-p)}\right)^{\frac{1}{q}+\frac{p-1}{p}} \frac{\left(\int_{0}^{\infty} v(\rho)^{q} \rho^{\frac{p q}{q-p}-1} d \rho\right)^{\frac{1}{q}}}{\left(\int_{0}^{\infty}\left|v^{\prime}(\rho)\right|^{p} \rho^{\frac{p q}{q-p}-1} d \rho\right)^{\frac{1}{p}}} \\
& =n^{\frac{1}{q}-\frac{1}{p}} \omega_{n}^{-\frac{1}{n}}\left(\frac{p^{2}}{(q-p)(n-p)}\right)^{\frac{1}{q}+\frac{p-1}{p}} I_{m, p}(v) \\
& \leq n^{\frac{1}{q}-\frac{1}{p}} \omega_{n}^{-\frac{1}{n}}\left(\frac{p^{2}}{(q-p)(n-p)}\right)^{\frac{1}{q}+\frac{p-1}{p}} I_{m, p}(\varphi) \\
& =n^{\frac{1}{q}-\frac{1}{p}} \omega_{n}^{-\frac{1}{n}} p^{\frac{1}{q}} q^{-\frac{1}{p}}(q-p)^{\frac{1}{p}-\frac{1}{q}} \frac{(p-1)^{1+\frac{1}{q}-\frac{2}{p}}}{(n-p)^{\frac{1}{q}-\frac{1}{p}+1}}\left[B\left(\frac{q}{q-p}, \frac{q(p-1)}{q-p}\right)\right]^{-\frac{1}{p}+\frac{1}{q}} \\
& =S_{n, p^{*}, q}
\end{aligned}
$$

The constant $S_{n, p^{*}, q}$ is achieved by any function of the form

$$
\psi(x)=\left(a+b|x|^{\frac{(n-p)(q-p)}{p(p-1)}}\right)^{-\frac{p}{q-p}}
$$

which solves the differential equation

$$
\left(r^{n-1}\left|u^{\prime}\right|^{p-1} \operatorname{sgn}\left(u^{\prime}\right)\right)^{\prime}=\frac{q}{p} \frac{(n-q)^{p}}{(p-1)^{p-1}} a b^{p-1} r^{-n+q \frac{n-p}{p}}|u|^{q-1}
$$

subject to the following conditions

$$
\int_{0}^{\infty} r^{\frac{p q}{q-p}-1}\left|u^{\prime}(r)\right|^{p} d r<\infty, u(r) \rightarrow 0 \text { as } r \rightarrow+\infty, u^{\prime}(r)=\mathrm{o}\left(r^{-\frac{q}{q-p}}\right) \text { as } r \rightarrow 0 \text { or }+\infty
$$

Clearly, as $q=p^{*}$ we recover the Talenti constant (2).

\subsection{Connections with Interpolation Inequalities with Weights}

As mentioned in the introduction, there is a natural relationship between Lorentz spaces and weighted Lebesgue spaces. Indeed, let $u$ be a radially decreasing function i.e. $u=u^{\sharp}$, then

$$
\begin{aligned}
\|u\|_{p, q}^{q} & =\int_{0}^{+\infty}\left[u^{*} t^{1 / p}\right]^{q} \frac{d t}{t} \\
& =n \omega_{n}^{\frac{q}{p}} \int_{0}^{\infty} u^{q} r^{q n / p} \frac{d r}{r} \\
& =n \omega_{n}^{\frac{q}{p}-1} \int_{\mathbb{R}^{n}}|u|^{q}|x|^{-\frac{n}{p}(p-q)} d x=n \omega_{n}^{\frac{q}{p}-1}\|u\|_{q,|x|^{-\frac{n}{p}(p-q)}}^{q}
\end{aligned}
$$


where $\|\cdot\|_{q,|x|^{-s}}$ denotes the norm of the weighted Lebesgue space $L^{q}\left(|x|^{-s} d x\right)$. If the weight $|x|^{-\frac{n}{p}(p-q)}$ is radially non increasing, and thus $p \geq q$, then thanks to the Hardy-Littlewood inequality,

$$
\begin{aligned}
\|u\|_{p, q}^{q} & =\int_{0}^{+\infty}\left[u^{*} t^{1 / p}\right]^{q} \frac{d t}{t} \\
& =n \omega_{n}^{\frac{q}{p}} \int_{0}^{\infty}\left(u^{\sharp}\right)^{q} r^{q n / p} \frac{d r}{r} \\
& =n \omega_{n}^{\frac{q}{p}-1} \int_{\mathbb{R}^{n}}\left|u^{\sharp}\right|^{q}|x|^{-\frac{n}{p}(p-q)} d x \\
& \geq n \omega_{n}^{\frac{q}{p}-1} \int_{\mathbb{R}^{n}}|u|^{q}|x|^{-\frac{n}{p}(p-q)} d x=n \omega_{n}^{\frac{q}{p}-1}\|u\|_{q,|x|^{-\frac{n}{p}(p-q)}}^{q}
\end{aligned}
$$

for any $u \in L^{p, q}$, so that

$$
L^{p, q}\left(\mathbb{R}^{n}\right) \hookrightarrow L^{q}\left(\mathbb{R}^{n},|x|^{-\frac{n}{p}(p-q)} d x\right) \quad \text { if } \quad p \geq q
$$

with optimal embedding constant given by

$$
\|u\|_{q,|x|^{-\frac{n}{p}(p-q)}} \leq n^{-\frac{1}{q}} \omega_{n}^{\frac{1}{q}-\frac{1}{p}}\|u\|_{p, q}
$$

The same embedding holds for any radially symmetric domain $\Omega \subset \mathbb{R}^{n}$.

Let us now go back to the family of (critical) Sobolev-Lorentz embeddings

$$
\mathcal{D}^{1, p}\left(\mathbb{R}^{n}\right) \hookrightarrow L^{p^{*}, q}\left(\mathbb{R}^{n}\right), \quad p^{*}=\frac{n p}{n-p}, \quad q \geq p
$$

Replacing $p$ with $p^{*}$ in Eq. 39 we get the following embeddings

$$
L^{p^{*}, q}\left(\mathbb{R}^{n}\right) \hookrightarrow L^{q}\left(\mathbb{R}^{n},|x|^{-n+q \frac{n-p}{p}} d x\right) \quad \text { if } \quad p \leq q \leq p^{*}
$$

namely

$$
\|u\|_{q,|x|^{-n+q \frac{n-p}{p}}} \leq n^{-\frac{1}{q}} \omega_{n}^{\frac{1}{q}-\frac{1}{p^{*}}}\|u\|_{p^{*}, q}
$$

with optimal embedding constant. This embedding, combined with Theorem 3, yields as a byproduct

$$
\mathcal{D}^{1, p}\left(\mathbb{R}^{n}\right) \hookrightarrow L^{q}\left(\mathbb{R}^{n},|x|^{-n+q \frac{n-p}{p}} d x\right) \quad \text { if } \quad p \leq q \leq p^{*}
$$

that is,

$$
\|u\|_{q,|x|^{-n+q \frac{n-p}{p}}} \leq n^{-\frac{1}{q}} \omega_{n}^{\frac{1}{q}-\frac{1}{p^{*}}} S_{n, p^{*}, q}\|\nabla u\|_{p}
$$

where the optimal constant is achieved if $p<q \leq p^{*}$ by the extremals (37).

The embedding Eq. 40 is well known in the literature (see [6, 17] and [12]):

$$
\mathcal{D}^{1, p}\left(\mathbb{R}^{n}\right) \subset L^{p^{*}(s)}\left(\mathbb{R}^{n},|x|^{-s} d x\right) \quad \text { if and only if } s \geq 0, \text { with } p^{*}(s)=p \frac{n-s}{n-p}
$$


whereas, if $s<0$, the emeddings holds just for the radial part of $\mathcal{D}^{1, p}\left(\mathbb{R}^{n}\right)$. The optimal embedding constant is achieved by dilations (and also translations, if $s=0$ ) of the function

$$
\phi_{\lambda}(x)=\lambda^{\frac{n-p}{p(p-s)}}\left(\lambda+|x|^{\frac{p-s}{p-1}}\right)^{-\frac{n-p}{p-s}}
$$

which is solution of the Euler Lagrange equation

$$
\left\{\begin{array}{l}
-\Delta_{p} u=c(n, p, s)|x|^{-s}|u|^{p^{*}(s)-2} u \\
u \in \mathcal{D}^{1, p}\left(\mathbb{R}^{n}\right)
\end{array} \quad \text { where } \quad c(n, p, s)=\frac{(n-s)(n-p)^{p-1}}{(p-1)^{p-1}}\right.
$$

Setting

$$
s=n-q \frac{n-p}{p}, \quad p<q \leq p^{*}=\frac{n p}{n-p}
$$

we find

$$
\psi_{\lambda}(x)=\lambda^{\frac{1}{q-p}}\left(\lambda+|x|^{\frac{(n-p)(q-p)}{p(p-1)}}\right)^{-\frac{p}{q-p}}
$$

which are solutions of the problem

$$
\left\{\begin{array}{l}
-\Delta_{p} u=c(n, p, q)|x|^{-n+q \frac{n-p}{p}}|u|^{q-2} u \\
u \in \mathcal{D}^{1, p}\left(\mathbb{R}^{n}\right)
\end{array} \quad \text { where } \quad c(n, p, q)=\frac{q}{p} \frac{(n-p)^{p}}{(p-1)^{p-1}}\right.
$$

and thus Eq. 38.

\section{The Limiting Case $q=p$ : Proof of Theorem 4}

As recalled in the introduction, the case $q=p$ has been already investigated by Alvino in [2]. Following the approach developed so far, notice that formally Theorem 3 does not apply as the quantity $(q-p)$ vanishes. However, we will show that the constant $S_{n, p^{*}, q}$, defined by Eq. 15, admits a limit for $q \rightarrow p$, which coincides with the optimal constant $S_{n, p^{*}, p}$, namely

$$
\lim _{q \rightarrow p} S_{n, p^{*}, q}=S_{n, p^{*}, p}=\omega_{n}^{-\frac{1}{n}} \frac{p}{n-p}
$$

Let us work out the following limit

$$
\begin{aligned}
\lim _{p \rightarrow q} S_{n, p^{*}, q} & =\lim _{p \rightarrow q} n^{\frac{1}{q}-\frac{1}{p}} \omega_{n}^{-\frac{1}{n}} p^{\frac{1}{q}} q^{-\frac{1}{p}}(q-p)^{\frac{1}{p}-\frac{1}{q}} \frac{(p-1)^{1+\frac{1}{q}-\frac{2}{p}}}{(n-p)^{\frac{1}{q}-\frac{1}{p}+1}}\left[B\left(\frac{q}{q-p}, \frac{q(p-1)}{q-p}\right)\right]^{-\frac{1}{p}+\frac{1}{q}} \\
& =\omega_{n}^{-\frac{1}{n}} \frac{(p-1)^{1-\frac{1}{p}}}{(n-p)} \lim _{p \rightarrow q} e^{\frac{q-p}{p q} \log (q-p)} \lim _{p \rightarrow q}\left[B\left(\frac{q}{q-p}, \frac{q(p-1)}{q-p}\right)\right]^{-\frac{1}{p}+\frac{1}{q}} \\
& =\omega_{n}^{-\frac{1}{n}} \frac{(p-1)^{1-\frac{1}{p}}}{(n-p)} \lim _{p \rightarrow q}\left[B\left(\frac{q}{q-p}, \frac{q(p-1)}{q-p}\right)\right]^{-\frac{1}{p}+\frac{1}{q}}
\end{aligned}
$$


since $\lim _{p \rightarrow q} \frac{q-p}{p q} \log (q-p)=0$. Now recall that the Beta and Gamma functions are related by

$$
B(a, b)=\frac{\Gamma(a) \Gamma(b)}{\Gamma(a+b)},
$$

so that

$$
B\left(\frac{q}{q-p}, \frac{q(p-1)}{q-p}\right)=\frac{\Gamma\left(\frac{q}{q-p}\right) \Gamma\left(\frac{q(p-1)}{q-p}\right)}{\Gamma\left(\frac{q p}{q-p}\right)}
$$

Furthermore, by Stirling's formula one has

$$
\Gamma(z)=z^{z} e^{-z}\left\{\sqrt{\frac{2 \pi}{z}}+\mathrm{O}\left(z^{-\frac{3}{2}}\right)\right\}, \quad \text { as } \quad \operatorname{Re} z \rightarrow+\infty
$$

from which we get the following estimates:

$$
\begin{gathered}
\Gamma\left(\frac{q}{q-p}\right)=\left(\frac{q}{q-p}\right)^{\frac{q}{q-p}} e^{-\frac{q}{q-p}}\left\{\sqrt{2 \pi \frac{q-p}{q}}+\mathrm{O}\left((q-p)^{\frac{3}{2}}\right)\right\}, \quad \text { as } \quad q \rightarrow p \\
\Gamma\left(\frac{q(p-1)}{q-p}\right)=\left(\frac{q(p-1)}{q-p}\right)^{\frac{q(p-1)}{q-p}} e^{-\frac{q(p-1)}{q-p}}\left\{\sqrt{2 \pi \frac{q-p}{q(p-1)}}+\mathrm{O}\left((q-p)^{\frac{3}{2}}\right)\right\}, \quad \text { as } \quad q \rightarrow p \\
\Gamma\left(\frac{q p}{q-p}\right)=\left(\frac{q p}{q-p}\right)^{\frac{q p}{q-p}} e^{-\frac{q p}{q-p}}\left\{\sqrt{2 \pi \frac{q-p}{q p}}+\mathrm{O}\left((q-p)^{\frac{3}{2}}\right)\right\}, \quad \text { as } \quad q \rightarrow p
\end{gathered}
$$

Therefore, as $q \rightarrow p$ we have

$$
\begin{aligned}
{\left[B\left(\frac{q}{q-p}, \frac{q(p-1)}{q-p}\right)\right]^{-\frac{1}{p}+\frac{1}{q}}=} & {\left[\frac{\Gamma\left(\frac{q}{q-p}\right) \Gamma\left(\frac{q(p-1)}{q-p}\right)}{\Gamma\left(\frac{q p}{q-p}\right)}\right]^{-\frac{q-p}{q p}} } \\
= & \left(\frac{q}{q-p}\right)^{-\frac{1}{p}} e^{\frac{1}{p}}\left\{\sqrt{2 \pi \frac{q-p}{q}}+\mathrm{O}\left((q-p)^{\frac{3}{2}}\right)\right\}^{-\frac{q-p}{q p}} \\
& \cdot\left(\frac{q(p-1)}{q-p}\right)^{-\frac{p-1}{p}} e^{\frac{p-1}{p}}\left\{\sqrt{2 \pi \frac{q-p}{q(p-1)}}+\mathrm{O}\left((q-p)^{\frac{3}{2}}\right)\right\}^{-\frac{q-p}{q p}} \\
& \cdot\left(\frac{q p}{q-p}\right) e^{-1}\left\{\sqrt{\left.2 \pi \frac{q-p}{q p}+\mathrm{O}\left((q-p)^{\frac{3}{2}}\right)\right\}^{\frac{q-p}{q p}}}\right. \\
= & q^{-\frac{1}{p}-\frac{p-1}{p}+1} p(p-1)^{-\frac{p-1}{p}}(q-p)^{\frac{1}{p}+\frac{p-1}{p}-1} e^{\frac{1}{p}+\frac{p-1}{p}-1} \\
& \times\left\{\sqrt{\left.\frac{2 \pi p(q-p)}{q(p-1)}+\mathrm{O}\left((q-p)^{\frac{3}{2}}\right)\right\}^{-\frac{q-p}{q p}}}\right. \\
& \rightarrow p(p-1)^{-\frac{p-1}{p}}, \quad \text { as } q \rightarrow p
\end{aligned}
$$


We conclude by inserting this value into Eq. 41 to obtain

$$
\begin{aligned}
\lim _{p \rightarrow q} S_{n, p^{*}, q} & =\omega_{n}^{-\frac{1}{n}} \frac{(p-1)^{1-\frac{1}{p}}}{(n-p)} \lim _{p \rightarrow q}\left[B\left(\frac{q}{q-p}, \frac{q(p-1)}{q-p}\right)\right]^{-\frac{1}{p}+\frac{1}{q}} \\
& =\omega_{n}^{-\frac{1}{n}} \frac{(p-1)^{1-\frac{1}{p}}}{(n-p)} p(p-1)^{-\frac{p-1}{p}} \\
& =\omega_{n}^{-\frac{1}{n}} \frac{p}{(n-p)}=S_{n, p^{*}, p}
\end{aligned}
$$

\section{References}

1. Adimurthy, Tintarev, K.: On a version of Trudinger-Moser inequality with Möbius shift invariance. Calc. Var. Partial Differ. Equ. 39, 203-212 (2010)

2. Alvino, A.: Sulla diseguaglianza di Sobolev in spazi di Lorentz. Boll. Un. Mat. Ital. A 14, 148-156 (1977)

3. Aubin, T.: Problème isopérimétrique et espaces de Sobolev. J. Differ. Geom. 11, 573-598 (1976)

4. Bennett, C., Rudnick, K.: On Lorentz-Zygmund spaces. Diss. Math. 175, 67 (1980)

5. Bennett, C., Sharpley, R.: Interpolation of operators. Pure and Applied Mathematics, vol. 129. Boston Academic Press, Inc. (1988)

6. Caffarelli, L., Kohn, R., Nirenberg, L.: First order interpolation inequalities with weights. Compos. Math. 53, 259-275 (1984)

7. Cassani, D., Ruf, B., Tarsi, C.: Best constants for Moser type inequalities in Zygmund spaces. Mat. Contemp. 36, 79-90 (2009)

8. Cassani, D., Ruf, B., Tarsi, C.: Group invariance and Pohozaev identity in Moser type inequalities. Comm. Contemp. Math. (2012). doi:10.1142/S021919971250054X

9. Curbera, G.P., Ricker, W.J.: Compactness properties of Sobolev imbeddings for rearrangement invariant norms. Trans. Amer. Math. Soc. 359, 1471-1484 (2007)

10. de Figueiredo, D.G., Ruf, B.: On a superlinear Sturm-Liouville equation and a related bouncing problem. J. Reine Angew. Math. 421, 1-22 (1991)

11. Edmunds, D.E., Kerman, R., Pick, L.: Optimal Sobolev Imbeddings involving rearrangementinvariant quasinorms. J. Funct. Anal. 170, 307-355 (2000)

12. Egnell, H.: Elliptic boundary value problems with singular coefficients and critical nonlinearities. Indiana Univ. Math. J. 38, 235-251 (1989)

13. Gidas, B., Ni, W.M., Nirenberg, L.: Symmetry and related properties via the maximum principle. Comm. Math. Phys. 68, 209-243 (1979)

14. Kerman, R., Pick, L.: Compactness of Sobolev imbeddings involving rearrangement-invariant norms. Studia Math. 186, 127-160 (2008)

15. Lions, P.L.: The concentration-compactness principle in the calculus of variations. The limit case I. Rev. Mat. Iberoam. 1, 145-201 (1985)

16. Maly, J., Pick, L.: An elementary proof of sharp Sobolev embeddings. Proc. Amer. Math. Soc. 130, 555-563 (2002)

17. Maz'ya, V.: Sobolev Spaces. Springer-Verlag, Berlin (1985)

18. Moser, J.: A sharp form of an inequality by Trudinger, N. Indiana Univ. Math. J. 20, 1077-1092 $(1970 / 71)$

19. Peetre, J.: Espaces dinterpolation et théorème de Soboleff. Ann. Inst. Fourier 16, 279-317 (1966)

20. Pustylnik, E.: On compactness of Sobolev embeddings in rearrangement-invariant space. Forum Math. 18, 839-852 (2006)

21. Sobolev, S.L.: Applications of functional analysis in mathematical physics. Translations of Mathematical Monographs, American Mathematical Society, vol. 7. Providence (1963)

22. Solimini, S.: A note on compactness-type properties with respect to Lorentz norms of bounded subsets of a Sobolev space. Ann. Inst. H. Poincaré, Anal. Non Linéaire 12, 319-337 (1995) 
23. Struwe, M.: Variational Methods. Applications to Nonlinear Partial Differential Equations and Hamiltonian Systems, 2nd edn. Springer-Verlag, Berlin (1996)

24. Talenti, G.: Best constant in Sobolev inequality. Ann. Mat. Pura Appl. 110, 353-372 (1976)

25. Tartar, L.: Imbedding theorems of Sobolev spaces into Lorentz spaces. Boll. Unione Mat. Ital. Sez. B Artic. Ric. Mat. 8(1), 479-500 (1998)

26. Yang, Y.: Solitons in Field Theory and Nonlinear Analysis, Springer Monographs in Mathematics. Springer-Verlag, New York (2001) 